\title{
Application of solid waste containing lead for gamma ray shielding material
}

\author{
Kh. REZAEE EBRAHIM SARAEE ${ }^{1 *}$, S. POURAJAM BAFERANI $^{1}$, O. TAHMASEBI $^{1}$ \\ ${ }^{I}$ Department of Nuclear Engineering, Faculty of Advance Sciences and Technologies, University of Isfahan, Isfahan,
}

Iran

Received: 01.02.2015; Accepted: 05.05.2015

\begin{abstract}
The basic strategies to decrease solid waste disposal problems have focused on the reduction of waste production and recovery of usable materials using waste and making raw materials. Generally, various materials have been used for radiation shielding in different areas and situations. In this study, a novel shielding material produced by a metallurgical solid waste containing lead has been analyzed in order to make a shielding material against gamma radiation. The photon total mass attenuation coefficients $(\mu / \rho)$ of the samples were measured experimentally and were calculated using WinXCom computer code, then theoretical and experimental values of total mass attenuation coefficient of each studied sample were compared with one another. Consequently, new shielding material made of solid waste containing lead is preferable as a shielding material against gamma radiation for buildings instead of concrete. Another goal of the paper is to obtain the optimum ratio of waste containing lead to achieve a balance between compressive strength and total mass attenuation coefficient. For this purpose an artificial neural network and genetic algorithm were used.
\end{abstract}

Keywords: Ceramic, Concrete, Gamma ray, Lead, Shielding, Solid waste

\section{INTRODUCTION}

In the last few decades, there has been a rapid increase in the waste materials and byproducts production due to population growth exponential rate, industry and technology development and consumerism growth (Alwaeli et al., 2012). No beneficial use is found for most of the waste and it leads to a waste disposal crisis, and contributes to health and environmental problems. Recycling of industrial waste is a logical option to manage this problem (Alwaeli, 2013). However, some of this waste can be used as substitute of raw materials in the construction industry. One type of such waste is solid waste containing lead from lead mines slags. Concrete and ceramic are widely used as building materials in the construction industry. Usage of waste as a partial replacement of raw materials in concrete and ceramic construction not only produces some products with better properties and eliminates or delays waste stream entry to the environment but also reduces the extraction of natural resources. So it has advantages such as omitting the cost of dumping, concrete and ceramic, minimizing environmental pollution problems and conservation of natural resources (Rajamane et al., 2003). The increasing use of radiation in different fields brings up the necessity to protect people against ionized radiation. Sample buildings in laboratories where radiation exists or nuclear waste deposition is buried can use materials which have more protection properties. There are several factors that influence the selection and use of radiation shielding materials. Taking into considerations, some factors, such as attenuation effectiveness, strength, resistance to damage, thermal properties, and cost efficiency can affect radiation protection in numerous ways. For example, metals are strong and resistant to radiation damage, but they undergo changes in their mechanical properties and are expensive. Likewise, concrete is strong, durable, and relatively inexpensive to produce, but it becomes weaker at elevated temperatures and less effective at blocking gamma rays. Therefore, the good shielding material should have adequate

*Corresponding author. Kh. Rezaee Ebrahim Saraee

Special Issue: The Second National Conference on Applied Research in Science and Technology

http://dergi.cumhuriyet.edu.tr/cumuscij (C2015 Faculty of Science, Cumhuriyet University 
mechanical properties and high mass attenuation coefficients (radiation absorption) and meanwhile irradiation effects on its mechanical and optical properties should be small.

Another proper method for experimental determination of mass attenuation coefficients is theoretical (Hubbell, 1982) or manual calculations using tabulated data that are generated using a computer program. For this purpose, a computer program (called XCOM) was developed to calculate cross-sections and attenuation coefficients for any element, compound or mixture, at energies from $1 \mathrm{keV}$ to $100 \mathrm{GeV}$ (Gerward et al., 2001; Gerward et al., 2004).

Neural networks and genetic algorithms demonstrate powerful problem solving capabilities. Genetic algorithms are global search methods that are based on principles such as selection, crossover and mutation. This paper aims to predict the optimum ratio of waste containing lead in order to achieve a balance between compressive strength and total mass attenuation coefficient. Although some research has focused on the effect of waste materials and byproducts on the properties of concrete, but there has been little research concerning the incorporation of solid waste containing lead extracted from mines in concrete and ceramic technology. This research was performed to evaluate the potential use of solid waste containing lead in concrete and ceramic production. The main goal of this study was to investigate the impact of lead industry solid waste on the compressive strength and absorption properties against different gamma energies (ranging from 661.615 to $1332.5 \mathrm{keV}$ ) using two different radioactive point sources $\left({ }^{137} \mathrm{Cs}\right.$ and $\left.{ }^{60} \mathrm{Co}\right)$ of concrete and ceramic.

\section{NUMERICAL METHODS}

\subsection{Specimen preparation}

In order to prepare shielding material, normal gray Portland cement (2 type) and a solid waste containing lead were used in the study. Gray Portland cement was obtained from Benvid gray cement Co. $25 \mathrm{~km}$ of BENVID Road in NAEIN, Iran. Solid waste containing lead was supplied from Nakhlak lead Complex Industry Inc. in Isfahan, Iran. The waste sample was dried at room temperature for 18 days prior to use, sieved by a 100 mesh standard sieve to obtain 100 mesh $(150 \mathrm{~mm})$ fraction sand then stored in a tightly closed jar throughout the study. Its chemical and mineralogical compositions were determined by Philips PW-2404 electron X-ray fluorescence spectroscopy. Chemical and mineralogical compositions showed that the waste sample contains $31.4 \% \mathrm{Si}, 18.11 \% \mathrm{Ca}, 16.16 \% \mathrm{~Pb}, 4.01 \% \mathrm{Ba}, 3.07 \mathrm{Mg}$ and $3.05 \% \mathrm{Al}$ as major elements. Varying amounts of other minor elements such as $\mathrm{S}, \mathrm{Fe}, \mathrm{Zn}, \mathrm{K}, \mathrm{Sr}, \mathrm{Na}, \mathrm{Ti}, \mathrm{Ce}, \mathrm{Cl}$, $\mathrm{Mn}, \mathrm{Cr}, \mathrm{P}, \mathrm{Cu}$ and $\mathrm{Zr}$ were also present in the material $\mathrm{X}$-ray fluorescence analysis (Table.1).

Shielding material was prepared by mixing cement or clay and the waste. For preparing concrete and ceramic, respectively cement and clay were replaced by waste having different proportions $(30 \%, 50 \%, 70 \%$, and $90 \%)$ based on the waste weight, in order to produce CemW30, CemW-50, CemW-70, and CerW-90 and CerW-30, CerW-50, CerW-70, and CerW-90. The mortar was then poured into cylindrical polyethylene moulds $(60 \mathrm{~mm}$ diameter and different heights in the range of 5 to $30 \mathrm{~mm}$ and was compacted. The samples were cured for 7 days in a controlled moisture chamber $(75 \% \mathrm{RH}) .1$ Cylindrical of mixed concrete and ceramic and 1 cylindrical of OC-0 were molded for compressive strength (Figure 1), and 4 cylinders of mixed concrete and ceramic and 4 cylinders of ordinary concrete and ceramic were cast for gamma radiation absorption measurements. Curing of all the concrete samples was done in water for 7 days. 
Application of solid waste containing lead for gamma ray shielding material

\subsection{Measurements}

Compressive strength was measured in accordance with ASTM Standards (1988c) ASTM C39/C39M-12a on cylindrical of $60 \mathrm{~mm}$ (diameter) $\times 120 \mathrm{~mm}$ (height). Tests were conducted on all the specimens after (7) days of curing. Average test results obtained in $\mathrm{Kg} / \mathrm{cm} 2$ are given in Table 2. Six different thicknesses $(0.5,1,1.5,2$ and $5 \mathrm{~cm})$ of radiation shielding materials specimens produced by the solid waste and ordinary gray Portland cement were prepared. The mass attenuation coefficients $(\mu / \rho)$ were determined by measuring the transmission of $\gamma$-rays using targets of those six different thicknesses individually. The experiments were performed using a low level gamma counting spectrometer including a $3 \mathrm{in} \times 5 \mathrm{in} \times 16 \mathrm{in} \mathrm{NaI}(\mathrm{TI})$ ORTEC Inc. detector, connected to a multichannel pulse height analyzer. The analysis of the collected spectra was carried out using DAS V2.3 the software package (ORTECInc.). The schematic view of the experimental setup is shown in Fig. 2. The detector was surrounded by lead sheet, shield to smooth the background $\gamma$-radiation. The sample was placed between the standard gamma point source and the detector. The experiment was repeated with and without the sample for $300 \mathrm{~s}$. The samples were irradiated by photons emitted from $60 \mathrm{Co}$ and $137 \mathrm{Cs}$ radioactive point sources in the range of 661.615 to $1332.5 \mathrm{keV}$. For each energy, the measurements were repeated six times for all the samples. Theoretical calculation of mass attenuation coefficients of the concrete and novel samples was carried out using WinXCom software and then, the obtained values were compared.

\section{RESULT AND DISCUSSION}

\subsection{Effect of solid waste containing lead substitute on the compressive strength}

As shown in Table 2, when the ratio of waste containing lead is increased, the compressive strength decreases in the mixed concrete and ceramic. The compressive strength of concrete made of $30 \%, 50 \%, 70 \%$, and $90 \%$ waste containing lead decreased by $73 \%, 58 \%, 29 \%$ and $14 \%$, respectively, compared with the ordinary concrete specimen. It also decreased by $83 \%$, $77 \%, 72 \%$ and $61 \%$ respectively, for mixed ceramic.

\subsection{Effect of solid waste containing lead substitute on the gamma radiation attenuation}

The total mass attenuation coefficients $(\mu / \rho)$ for concrete and the new materials have been measured and calculated using the WinXCom software at energies from $10 \mathrm{keV}$ to $5 \mathrm{MeV}$, which is in the border of experimental studied energies $(661.615 \mathrm{keV}-1332.5 \mathrm{keV})$. Compound composition of novel shielding materials and chemical analysis of clay and concrete is shown in Table 1. Oxygen, calcium, silicon and lead approximately are higher constituents than others. Total experimental and theoretical mass attenuation coefficients of each studied sample were compared with one another (Table. 3). It showed that the calculated and measured values are in a good agreement. The experimental total mass attenuation coefficients decrease with photon energy increase for the new clay and concrete materials. Therefore, photoelectric effect and Compton scattering are dominant. In the high energy, the total mass attenuation coefficient smoothly decreases in materials with the increase of photon energy. The Compton scattering is a dominant reaction and its cross-section may be considered as the main interaction. Nevertheless, total mass attenuation coefficients are almost the same for the three samples in high energy. In the low energy region, the calculated total mass attenuation coefficients sharply decrease for all the samples. These sharp decreases have continued through the low energy region and they slightly decrease afterwards. Additionally, the total mass attenuation coefficient difference of three studied samples, especially new material and concrete, has been clearly seen in the low energy region (Fig. 3) and this may be attributed to the fact that the photoelectric effects are distinct due to different substance compositions of new material and concrete. As should be expected, density is the main parameter which affects the mass attenuation coefficient. 
However, in the present work, it has been clearly seen that variations in the chemical composition of the materials are more significant. Despite the ceramic $(50 \%)$ has a lower density $\left(0.52 \mathrm{gcm}^{-3}\right)$ than concrete $(50 \%)$, the total mass attenuation coefficient is approximately higher than concrete (50\%) which has been measured and calculated in the low and high energy region. It is clear from table 3 that the linear attenuation coefficient of gamma radiation of a concrete specimen increases significantly with the increase of solid waste containing lead content in the concrete and ceramic mixes. The linear attenuation coefficient of concrete mixes made of $30 \%, 50 \%, 70 \%$, and $90 \%$ waste containing lead increased by $1.24 \%, 2.14 \%, 2.27 \%$, and $2.34 \%$, respectively, compared with the ordinary concrete specimen. It also increased in ceramic by $1.54 \%, 1.88 \%, 1.90 \%$, and $2.08 \%$, respectively. However, as mentioned above, the compressive strength decreases. Then algorithm genetic was used to optimize content of solid waste containing lead in the specimens. The network has a two hidden layer with 5 perceptron in each layer with 1 input and 2 outputs (1-5-5-2). Activation function was tangent sigmoid for all layers. Training was performed with error back propagation algorithm with 87 epochs. The multi objective genetic algorithm was used for optimization after 201 iterations. The results of multi objective GA are represented in Table 4.

\section{CONCLUSION}

A new shielding material was made using a solid waste containing lead in this study. The mass attenuation coefficients $(\mu / \rho)$ and the compressive strength of the material show that the novel shielding material made from a solid waste containing lead would be preferred as a shielding material for buildings against gamma radiation.

\section{REFERENCES}

[1] Alwaeli, M., Nadziakiewicz, J., Recycling of scale and steel chip waste as a partial replacement of sand in concrete, Construction and Building Materials 28 (2012) 157-163.

[2] Alwaeli, M., Application of granulated lead-zinc slag in concrete as an opportunity to save natural resources, Radiation Physics and Chemistry 83 (2013) 54-60.

[3] Gerward, L., Guilbert, N., Jensen, K.B., Levring, H., 2001.X-ray absorption in matter. Re engineering XCOM. Radiat.Phys.Chem.60, 23-24.

[4] Gerward, L., Guilbert, N., Jensen, K.B., Levring, H., 2004.WinXCom a program for calculating X-ray attenuation coefficients. Radiat.Phys.Chem.71, 653-654.

[5] Hubbell, J.H., 1982. Photon mass attenuation and energy absorption coefficients from $1 \mathrm{keV}$ to $20 \mathrm{MeV}$. Int. J. Appl. Radiat. Isot. 33, 1269-1290.

[6] Rajamane, N.P., Peter, J.A., Dattatreya, J.K., Neelamegam, M., Gopalakrishnan, S., 2003. Improvement in properties of high performance concrete with partial replace- ment of cement by ground granulated blast furnace slag. J. Inst. Eng. 84, 38-42. 